\title{
Mortality Among Veterans with Major Mental Illnesses Seen in Primary Care: Results of a National Study of Veteran Deaths
}

\author{
Ranak B. Trivedi, $P h D^{1,2}$, Edward P. Post, $M D, P h D^{3,4}$, Rebecca Piegari, $M S^{5}$, \\ Joseph Simonetti, MD, MPH ${ }^{6,7,8}$, Edward J. Boyko, MD ${ }^{9,10}$, Steven M. Asch, MD, MPH ${ }^{1,11}$, \\ Alaina Mori, BA ${ }^{12}$, Bruce A. Arnow, $\mathrm{PhD}^{13}$, Stephan D. Fihn, MD, MPH ${ }^{14,15}$, \\ Karin M. Nelson, MD, MS, $H S^{12,14}$, and Charles Maynard, $P h D^{8,16}$
}

\begin{abstract}
${ }^{1}$ Center for Innovation to Implementation, VA Palo Alto Health Care System 795 Willow Rd 152-MPD, Menlo Park, CA, USA; ${ }^{2}$ Division of Public Mental Health and Population Sciences, Department of Psychiatry and Behavioral Sciences, Stanford University, Stanford, CA, USA; ${ }^{3}$ Center for Clinical Management Research, VA Ann Arbor Health Care System, Ann Arbor, MI, USA; ${ }^{4}$ Department of Internal Medicine, University of Michigan School of Medicine, Ann Arbor, MI, USA; 5 Office of Clinical Systems Development and Evaluation, Canandaigua VA Medical Center, Canandaigua, NY, USA; ${ }^{\circ}$ Rocky Mountain Mental Illness Research, Education and Clinical Center, Rocky Mountain Regional VA Medical Center, Aurora, CO, USA; ${ }^{7}$ Division of Hospital Medicine, University of Colorado Anschutz School of Medicine, Aurora, CO, USA; ${ }^{8}$ Denver-Seattle Center of Innovation for Veteran-Centered and Value-Driven Care, Seattle, WA, USA; ${ }^{2}$ Seattle Epidemiologic Research and Information Center, VA Puget Sound Health Care System, Tacoma, WA, USA; ${ }^{10}$ Department of Epidemiology, University of Washington School of Public Health, Seattle, WA, USA; " ${ }^{1}$ Department of General Internal Medicine, Stanford University, Stanford, CA, USA; ${ }^{12}$ Primary Care Analytics Team, VA Puget Sound Health Care System, Tacoma, WA, USA; ${ }^{13}$ Department of Psychiatry and Behavioral Sciences, Stanford University, Stanford, CA, USA; ${ }^{14}$ General Internal Medicine, University of Washington, Seattle, WA, USA; ${ }^{15}$ Division of General Internal Medicine, Harborview Medical Center, Seattle, WA, USA; ${ }^{16}$ Department of Health Services, University of Washington, Seattle, WA, USA.
\end{abstract}

BACKGROUND: Premature mortality observed among the mentally ill is largely attributable to chronic illnesses. Veterans seen within Veterans Affairs (VA) have a higher prevalence of mental illness than the general population but there is limited investigation into the common causes of death of Veterans with mental illnesses.

OBJECTIVE: To characterize the life expectancy of mentally ill Veterans seen in VA primary care, and to determine the most death rates of combinations of mental illnesses. DESIGN: Retrospective cohort study of decedents.

SETTING/PARTICIPANTS: Veterans seen in VA primary care clinics between 2000 and 2011 were included. Records from the VA Corporate Data Warehouse (CDW) were merged with death information from the National Death Index.

MAIN MEASURES: Mental illnesses were determined using ICD9 codes. Direct standardization methods were used to calculate age-adjusted gender and cause-specific death rates per 1000 deaths for patients with and without depression, anxiety, post-traumatic stress disorder (PTSD), substance use disorder (SUD), serious mental illness (SMI), and combinations of those diagnoses.

KEY RESULTS: Of the 1,763,982 death records for Veterans with $1+$ primary care visit, 556,489 had at least one

Preliminary results from this study have been previously presented at the annual meeting of the Society of General Internal Medicine and the Annual Research Meeting of Academy Health in 2017.

Electronic supplementary material The online version of this article (https://doi.org/10.1007/s11606-019-05307-w) contains supplementary material, which is available to authorized users. mental illness. Heart disease and cancer were the two leading causes of death among Veterans with or without a mental illness, accounting for approximately 1 in 4 deaths. Those with SUD ( $n=204,950)$ had the lowest mean age at time of death (64 \pm 12 years). Among men, the death rates were as follows: SUD (55.9/1000); anxiety (49.1/1000); depression (45.1/1000); SMI (40.3/1000); and PTSD (26.2/1000). Among women, death rates were as follows: SUD (55.8/1000); anxiety (36.7/1000); depression (45.1/1000); SMI (32.6/1000); and PTSD (23.1/1000 deaths). Compared to men (10.8/1000) and women $(8.7 / 1000)$ without a mental illness, these rates were multiple-fold higher in men and in women with a mental illness. A greater number of mental illness diagnoses was associated with higher death rates among men and women $(p<0.0001)$.

CONCLUSIONS: Veterans with mental illnesses, particularly those with SUD, and those with multiple diagnoses, had shorter life expectancy than those without a mental illness. Future studies should examine both patient and systemic sources of disparities in providing chronic illness care to Veterans with a mental illness.

KEY WORDS: mental disorders; cause of death; Veterans; primary care medical home.

J Gen Intern Med 35(1):112=8

DOI: $10.1007 / \mathrm{s} 11606-019-05307-\mathrm{w}$

(c) Society of General Internal Medicine (This is a U.S. government work and not under copyright protection in the U.S.; foreign copyright protection may apply) 2019 
$\mathrm{O}$ ver the last decade, mental illnesses have been recognized as important contributors to premature mortality. ${ }^{1} \mathrm{~A}$ recent meta-analysis of 203 studies from 29 countries estimated allcause mortality across all mental illnesses and found that 8 million people die globally due to mental illness, and that mental illnesses led to a median reduction in life expectancy of 10 years. ${ }^{2}$

Prior studies attempting to explain the shorter life expectancy among those with mental illness have found that such patients largely die of medical causes such as diabetes and heart disease. ${ }^{3}$ Olfson and colleagues (2015) found that patients with schizophrenia were 3.5 times more likely to die than the general population, due to largely preventable causes such as high blood pressure, high cholesterol, and diabetes. ${ }^{4,5}$ However, those studies, such as those conducted using the National Health Interview Survey data, relied on self-report for mental illness diagnoses, medical diagnoses, and whether or not they were receiving treatment. ${ }^{6}$ Many of these studies did not have sufficient sample size to examine or compare the life expectancy of individuals with different mental illnesses or combinations of illness. Mental illnesses are frequently comorbid, and assessing the cumulative effects of mental illness on mortality is critical to identifying highest risk groups. ${ }^{7}$ The widely cited Case and Deaton (2015) study highlighted a rise in middle-aged mortality in the USA attributable to drug and alcohol poisoning, especially among the poorly educated, ${ }^{8}$ suggesting that substance use disorders (SUD) may be especially important in understanding the effects of mental illness on mortality.

Understanding the life expectancy and common causes of death among the mentally ill has the potential of reducing health disparities. Health disparities among those with a mental health condition are introduced in important ways. For the so-called natural causes of death, treating high blood pressure, elevated cholesterol, and/or diabetes could go a long way toward increasing life expectancy in individuals with mental health conditions. There is ample evidence that those with a mental health condition are less likely to receive primary prevention and guideline concordant treatment for these conditions. ${ }^{9}$ For external causes of death including suicide and unintentional injury deaths, knowing who is more likely to die of these causes could lead to interventions which could prevent premature deaths. This is particularly applicable to individuals with SUD; providing appropriate treatment for alcohol use disorders could result in reduction of alcohol use which is associated with higher levels of motor vehicle collision deaths.

The Veterans Health Administration within the Department of Veterans Affairs (VA) is one of the largest integrated healthcare system in the USA, and provides care to nearly 10 million Veterans. Veterans seen in VA have poorer physical and mental health compared to the general population. ${ }^{10}$ One quarter of Veterans seen in the VA primary care have at least one mental illness. ${ }^{7}$ Zivin et al. (2015) showed that depression is associated with worse mortality than those without depression among Veterans. ${ }^{11}$ Furthermore, as was noted by Trivedi et al, those with SUD and SMI were at higher risk of mortality at 1 year than those without these diagnoses. ${ }^{7}$ The purpose of this study was to evaluate the life expectancy of Veterans with mental illness receiving primary care from the Veterans Health Administration. We had two further objectives: (1) to determine the common causes of death in Veterans with mental illness, and (2) to evaluate the combinations of mental illnesses which were associated with highest rate of death.

\section{METHODS}

Study Design. We conducted an observational cohort study of Veterans who were seen in primary care within the VA between 2000 and 2011. Consistent with other studies examining VA primary care, ${ }^{7,12}$ patients were identified by being in the primary care management module. That is, they were enrolled and assigned a primary care provider and had seen the primary care provider at least once in the prior 1 year. Veteran data were obtained from the VA Utilization Files included in the Corporate Data Warehouse (CDW). ${ }^{13}$ We used ICD9 codes from the prior 10 years to identify patients with depression, anxiety, post-traumatic stress disorder (PTSD), serious mental illness (SMI; bipolar disorder and/or schizophrenia), and/or SUD. ${ }^{7}$ SUD was diagnosed if the records showed a diagnosis of an SUD at either 1 outpatient visits or 1 inpatient visit in the prior year; the rest of the diagnoses were based on 2 outpatient visits or 1 inpatient visit in the prior year. This is because ICD-9 diagnosis codes for SUD have poor sensitivity compared to other mental illness codes or medical diagnosis codes. ${ }^{14}$ Tobacco use disorder was not included in the definition of SUD. These five groups were not mutually exclusive; for instance, individuals who had both depression and PTSD would be included in both groups. We created two more categories for comparison: one that included Veterans who had any of the 5 mental illnesses, and another that included Veterans who did not have any of the 5 mental illnesses. ICD9 codes used to determine the presence of mental illnesses are provided in online Appendix A.

CDW data were merged with data obtained from death records through the VA Department of Defense Suicide Data Repository (SDR), which identifies cause of death by linking Veterans to the National Death Index (NDI). ${ }^{15}$ The primary cause of death was noted using ICD10 codes, and date of death was abstracted from SDR records. ${ }^{16}$ A total of $1,766,472$ death records were linked with VA primary care patients using social security numbers. We excluded those with missing data $(<0.1 \%)$. The final file included $1,763,982$ individuals $(99.9 \%$ of the original file) who died between the years 2000 and 2011. The causes of death of most interest were most common conditions such as cardiovascular diseases, as well as those described in Case and Deaton (2015), ${ }^{8}$ and are provided in online Appendix B.

Statistical Analyses. We report the distributions of sociodemographic characteristics, mean life expectancy, and specific causes of death within each mental illness category. 
We conducted age-adjusted regression analyses to estimate death rates (cause-specific deaths per 1000 deaths) between 2000 and 2011 using the direct method of standardization separately for women and men, using the 2010 USA population as the standard. Since there were relatively few deaths in the years 2000 through 2002 ( $<3 \%$ of all deaths), we calculated age-adjusted rates for the years 2003 through 2011. Comorbidity of mental illnesses is often not considered in mental health research likely due to small numbers. Our sample size allowed us to examine different dyadic combinations and emphasize how combinations could increase the mortality risk compared to individual mental illnesses. We therefore computed age-adjusted rates for subgroups of interest which included SMI and/or SUD. We also examined the life expectancy of patients with $1,2,3,4$, or all 5 conditions. Life expectancy was the age at time of death derived from the National Death Index.

\section{RESULTS}

The sample was predominantly male $(98.1 \%)$ and White (86.7\%). Of the 1,763,982 included in the study, 556,489 (31.5\%) had at least one mental illness (Table 1). The most common mental illness was depression $(n=355,173,20.1 \%)$, followed by SUD $(n=204,950,11.6 \%)$, anxiety $(n=135,421$, $7.6 \%)$, PTSD $(n=96,641,5.4 \%)$, and SMI $(n=83,275$, $4.7 \%$ ). In the years $2000-2005,32.1 \%$ of deaths occurred among Veterans with no mental illness diagnosis. In the years 2006-2011, 67.9\% of deaths occurred among Veterans with no mental illness diagnosis. The reporting of death data became more stringent in 2005; therefore, data between 2000 and 2005 may not be as rigorous for the years 2006-2011. The mean age of Veterans without a mental illness was 78 years, compared to a mean age of 71 years for Veterans with at least one mental illness. All mental illnesses were associated with premature death. The shortest life expectancy was among patients with SUD (64 years), and SUD, SMI, and PTSD diagnoses were associated with shorter life by more than a decade on average, while depression and anxiety diagnoses were associated with shorter life by 6 years on average.

Table 2 shows unadjusted cause of death by mental illness diagnosis. Majority of the deaths occurred due to either heart disease or cancer, irrespective of having a mental illness diagnosis. The cause of death varied by mental illness categories. The percentage of deaths by accidents, alcohol-related liver disease, assault, and human immunodeficiency virus infection (HIV) is highest among those with SUD, and considerably higher than those without mental illness diagnosis. Notably, $6.5 \%$ of deaths among individuals with SUD were due to alcohol-related liver disease, compared to less than $1 \%$ of individuals without any mental illness. A greater proportion of PTSD (6.6\%), SUD (7.0\%), and SMI (7.1\%) deaths were deemed accidental compared to depression $(4.7 \%)$ and anxiety (5.2\%). Two to $3 \%$ of individuals died of suicide across all mental illness categories. Of the 13,881 individuals who died by suicide, $52 \%(n=7263)$ did not carry a diagnosis of mental illness. The proportion of individuals who died of heart disease, cancer, cerebrovascular disease, influenza, kidney disease, chronic respiratory disease, and septicemia were similar in the groups with or without a mental illness.

As can be seen across Figures 1, 2, and 3, the death rates across most mental illnesses remained stable over time, although the death rates among those with SUD appeared to decline since 2008. Figure 1a and $\mathrm{b}$ show age-adjusted death rates across the mental illness groups by men (1a) and women (1b). Men with SUD had an average death rate of 55.9/1000 deaths (across the study period) which was nearly five times the death rate of Veterans without a mental illness $(10.77 / 1000$ deaths). Both anxiety (49.07/1000 deaths) and depression (45.05/1000 deaths) had higher death rates than SMI (40.34/ 1000 deaths). Of the mental illness categories, PTSD had the lowest death rate albeit still higher than not having any mental illness (26.18/1000 deaths). Women with SUD had an average death rate of 55.82/1000 deaths (across the study period) which was nearly 6.5 times the death rate of Veterans without a mental illness (8.69/1000 deaths). Both anxiety (36.73/1000 deaths) and depression (45.05/1000 deaths) had higher death rates than SMI (32.63/1000 deaths). Similar to men, women with PTSD had the lowest death rate albeit still higher than not having any mental illness (23.10/1000 deaths).

As noted in Table 1, the number of women who met the study criteria was small over the 9 years making it difficult to obtain stable estimates for analyses that examined combinations of illnesses and the cumulative effects of multiple mental illnesses. Men with SUD and SMI experienced higher death rates when combined with any of the other mental illness categories, with the highest death rate being for individuals who carried diagnoses of depression, SUD, and SMI (Fig. 2). Both men (Fig. 3a) and women (Fig. 3b) with a greater number of mental illness diagnoses had a higher death rate, with those with 3 or more mental illnesses having the highest death rate $(p$ 's $<0.0001)$.

\section{DISCUSSION}

In this national sample of Veterans seen in VA primary care, we found that individuals with mental illnesses had earlier mortality and higher death rates than those without any mental illnesses. Individuals with SUD lived 14 fewer years and had a death rate higher than patients with no mental illness. We found that causes of death varied by mental illness. The highest proportion of alcohol-related liver disease was among Veterans diagnosed with a SUD while the highest proportion of suicide was among Veterans diagnosed with a SMI.

For both men and women, having a SUD was associated with several-fold increases in death rates across the 9-year study period. More mental illnesses led to higher death rates among men, although these analyses could not be conducted among women due to smaller numbers of decedents. Among men, 
Table 1 Baseline Sociodemographic Characteristics

\begin{tabular}{|c|c|c|c|c|c|c|c|}
\hline Variable & $\begin{array}{l}\text { No mental } \\
\text { illness }\end{array}$ & $\begin{array}{l}\text { Any mental } \\
\text { illness }\end{array}$ & Depression & PTSD $^{a}$ & $\begin{array}{l}\text { Substance } \\
\text { use disorder }\end{array}$ & Anxiety & $\begin{array}{l}\text { Serious mental } \\
\text { illness }\end{array}$ \\
\hline$N$ & $1,207,493$ & 556,489 & 355,173 & 96,641 & 204,950 & 135,421 & 83,275 \\
\hline Age, years $(\mathrm{M} \pm 1 \mathrm{SD})$ & $78 \pm 10$ & $71 \pm 13$ & $72 \pm 13$ & $67 \pm 13$ & $64 \pm 12$ & $72 \pm 13$ & $66 \pm 13$ \\
\hline Men $(\%)$ & 98.1 & 97.4 & 96.8 & 97.8 & 98.3 & 96.4 & 95.9 \\
\hline \multicolumn{8}{|l|}{ Race } \\
\hline White $(\%)$ & 86.7 & 81.5 & 84.2 & 77.5 & 72.2 & 87.7 & 74.8 \\
\hline African American (\%) & 8.9 & 12.2 & 9.6 & 13.9 & 20.3 & 6.9 & 17.2 \\
\hline Hispanic $(\%)$ & 2.6 & 3.8 & 3.7 & 4.4 & 4.4 & 3.0 & 4.9 \\
\hline Asian/Pacific Islander (\%) & 0.7 & 0.7 & 0.6 & 1.1 & 0.6 & 0.6 & 0.8 \\
\hline Native American (\%) & 0.4 & 0.6 & 0.5 & 1.1 & 0.8 & 0.5 & 0.6 \\
\hline Other & 0.2 & 0.1 & 0.1 & 0.2 & 0.1 & 0.1 & 0.1 \\
\hline Multiracial (\%) & 0.5 & 1.2 & 1.2 & 1.9 & 1.5 & 1.2 & 1.6 \\
\hline \multicolumn{8}{|l|}{ Death year } \\
\hline $2000-2005(\%)$ & 32.1 & 26.2 & 24.5 & 22.3 & 26.4 & 23.4 & 29.3 \\
\hline 2006-2011 (\%) & 67.9 & 73.8 & 75.5 & 77.7 & 73.6 & 76.6 & 70.7 \\
\hline
\end{tabular}

${ }^{a}$ PTSD post-traumatic stress disorder

${ }^{b}$ Serious mental illness includes diagnoses of schizophrenia and/or bipolar disorder

${ }^{c}$ Death year refers to the distribution of deaths by 2 categories of year of death. In the years 2000-2005, 32.1\% of deaths occurred for Veterans with no mental illness diagnosis. In the years 2006-2011, 67.9\% of deaths occurred

having SUD also increased the mortality rate of any other mental illness, and having depression, SUD, and SMI was associated with the highest morality rate. SUD is a national epidemic and its threat to life expectancy is perhaps not a surprise. Historically, those with SUD have been treated by specialists in mental health specialty settings, which have included both intensive outpatient and inpatient residential settings. However, these programs can be difficult to access and difficult to complete for the patients. There is an increasing focus on medication-assisted treatment (MAT) and medicationassisted recovery (MAR) that provide pharmacological interventions for patients with SUDs, such as disulfiram for alcohol use disorders and naltrexone for opioid use disorders. In light of the opioid use crisis, there has been a movement to equip not only primary care providers, but patients, first responders, and even citizens with naloxone to be used if an overdose is suspected. ${ }^{17}$ These approaches can improve the management of SUDs in primary care settings where providers can coordinate care along with chronic illness prevention and treatment.
Among individuals who died by suicide, over half had not been diagnosed with a mental illness. A similar finding was also reported recently by Boggs et al. using data from Kaiser Permanente. ${ }^{18}$ This raises important questions regarding the best strategy of monitoring patients for suicide risk, as the most common strategy involves targeting efforts toward those with a mental illness. The VA has made significant investments in improving the detection and prevention of suicide among Veterans. Foremost includes annual screening and developing educational resources for patients, their families, and providers, as well as resources for families who may have lost someone to suicide. The VA has also established a national center of excellence in suicide prevention that takes a public health approach to suicide prevention. The recent implementation of the Recovery Engagement and Coordination for Health-Veterans Enhanced Treatment (REACH-VET) is another example of such an effort. The REACH-VET program uses predictive analytics to identify high-risk Veterans from medical charts, who are then contacted by a mental health specialist or their clinician. Such

Table 2 Unadjusted Cause of Death by Mental Illness, \%

\begin{tabular}{|c|c|c|c|c|c|c|c|}
\hline Cause of death & $\begin{array}{l}\text { No mental } \\
\text { illness }\end{array}$ & $\begin{array}{l}\text { Any mental } \\
\text { illness }\end{array}$ & Depression & PTSD & $\begin{array}{l}\text { Substance use } \\
\text { disorder }\end{array}$ & Anxiety & $\begin{array}{l}\text { Serious mental } \\
\text { illness }\end{array}$ \\
\hline$N$ & $1,207,493$ & 556,489 & 355,173 & 96,641 & 204,950 & 135,421 & 83,275 \\
\hline Heart disease & 26.1 & 23.2 & 23.4 & 23.7 & 21.0 & 23.6 & 24.3 \\
\hline Cancer & 26.8 & 23.1 & 21.7 & 24.3 & 24.6 & 22.9 & 18.7 \\
\hline Cerebrovascular disease & 4.8 & 4.0 & 4.3 & 3.2 & 2.9 & 3.4 & 3.4 \\
\hline $\begin{array}{l}\text { Chronic lower respiratory } \\
\text { disease }\end{array}$ & 6.9 & 7.9 & 7.8 & 6.4 & 6.7 & 10.4 & 7.1 \\
\hline Accidental deaths & 2.4 & 4.6 & 4.7 & 6.6 & 7.0 & 5.2 & 7.1 \\
\hline Diabetes mellitus & 3.3 & 3.2 & 3.6 & 3.7 & 2.4 & 2.8 & 3.6 \\
\hline Influenza and pneumonia & 2.2 & 2.1 & 2.1 & 1.7 & 1.6 & 2.1 & 2.4 \\
\hline Kidney & 2.4 & 1.8 & 1.9 & 1.5 & 1.2 & 1.7 & 1.5 \\
\hline Suicide & 0.6 & 1.7 & 2.0 & 2.3 & 2.3 & 2.2 & 3.0 \\
\hline Alcoholic liver disease & 0.7 & 2.8 & 1.9 & 3.0 & 6.5 & 1.6 & 2.2 \\
\hline Septicemia & 1.4 & 1.5 & 1.4 & 1.4 & 1.5 & 1.4 & 1.6 \\
\hline Assault & 0.1 & 0.4 & 0.4 & 0.5 & 0.7 & 0.4 & 0.8 \\
\hline HIV $^{\mathrm{a}}$ & 0.1 & 0.5 & 0.5 & 0.4 & 0.9 & 0.3 & 0.7 \\
\hline Other & 22.2 & 23.3 & 24.4 & 21.2 & 20.7 & 22.1 & 23.6 \\
\hline
\end{tabular}

${ }^{a} H I V$ human immunodeficiency virus; PTSD post-traumatic stress disorder 

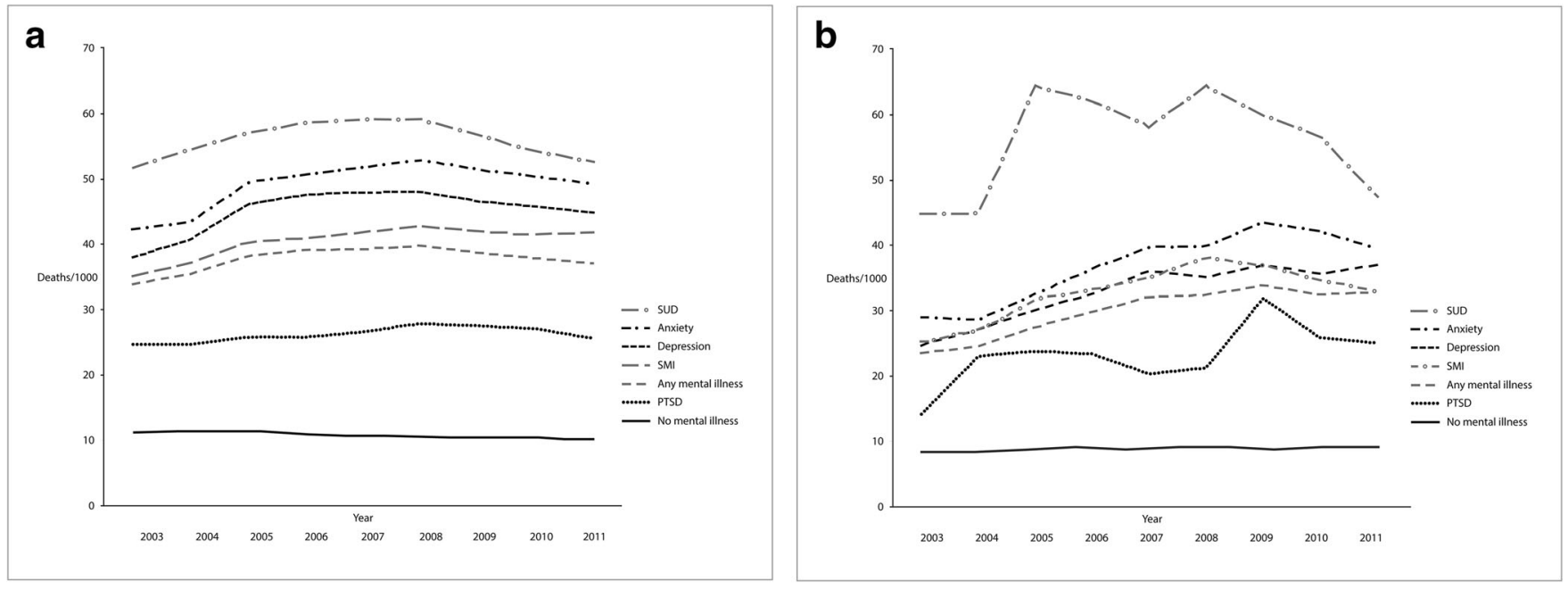

Figure 1 a Age-adjusted death rates by mental illness categories, men (PTSD, post-traumatic stress disorder; SUD, substance use disorder; SMI, serious mental illness). b Age-adjusted death rates by mental illness categories, women (PTSD, post-traumatic stress disorder; SUD, substance use disorder; SMI, serious mental illness)

concerted efforts by the VA across all aspects of healthcare delivery will be critical to preventing Veteran suicides.

A critical finding is that most of the patients with mental illness died of preventable causes such as ischemic heart disease. Prior studies have suggested that premature mortality is moderated by behavior and healthcare access, ${ }^{19}$ while other studies suggest that poor outcomes are moderated by physiology. ${ }^{1} \mathrm{~A}$ recent meta-analysis noted the paucity of behavioral intervention studies that target cardiovascular disease risk factors among patients with serious mental illness, despite the fact that these patients have a high prevalence of common risk factors such as obesity and hypercholesterolemia. ${ }^{20}$ Therefore, an important intervention to extend life among the mentally ill may be to ensure that all Veterans are receiving high-quality preventive

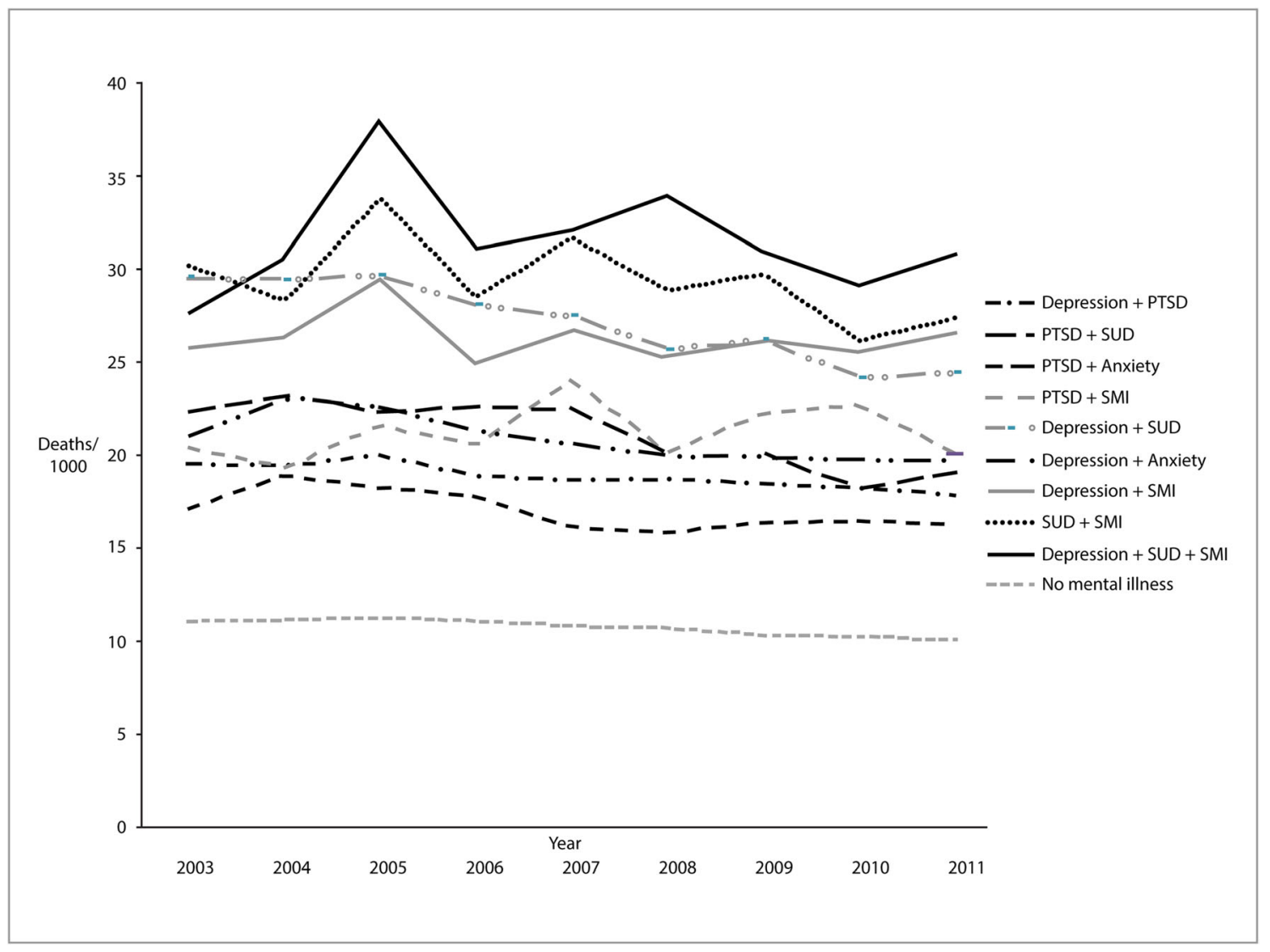

Figure 2 Age-adjusted death rates by combinations of mental illness, men 
a

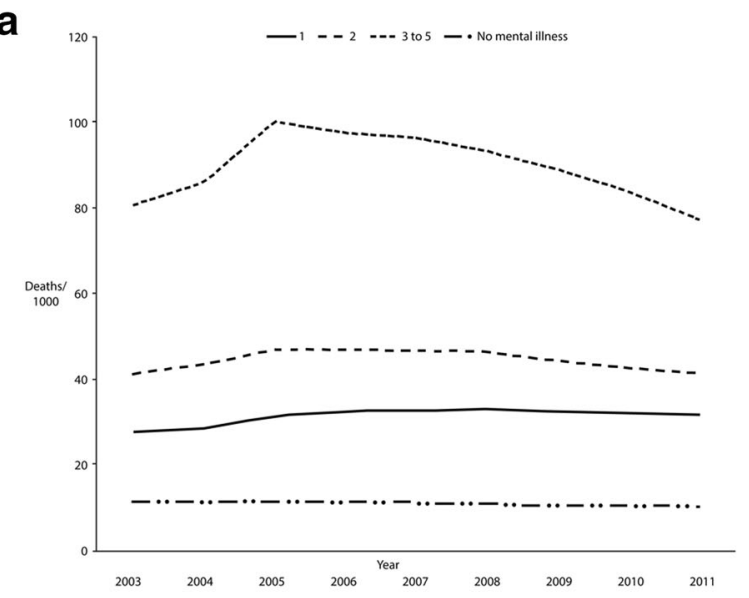

b

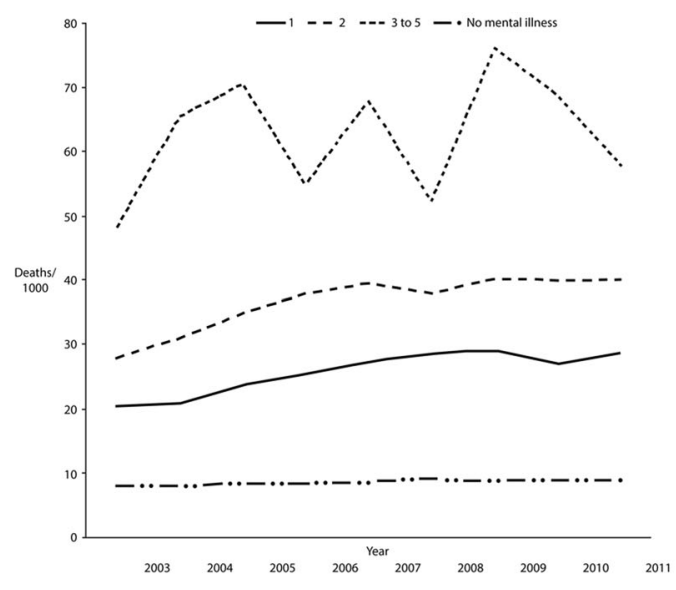

Figure 3 a Age-adjusted death rates by number of mental illnesses, men. b Age-adjusted death rates by number of mental illnesses, women

care through their primary care providers. The VA initiatives of patient-centered medical home ${ }^{12}$ and primary care and mental health integration ${ }^{21}$ are important strides in this direction.

Our study extends existing literature in several important ways. First, our sample size made it possible to examine combinations of various mental illness to identify the most lifelimiting combinations. Prior research has lacked sufficient sample size or other data that allow rigorous examination of multiple mental illnesses. Second, we use diagnostic codes from administrative data as compared with self-report of diagnosis or distress making our findings more relevant to clinical practice. Third, the availability of death records allowed us to determine primary causes of death. Prior research has evaluated mortality risk but less light has been shed on the causes of death. Fourth, the longitudinal data allowed us to evaluate changes in mortality rates over time which led to the recognition that death rates have stayed stable over time within each mental illness group.

Our study has important limitations. As with all VA studies, our sample was majority male and identified as non-Hispanic White. While this may limit generalizability to other populations, it is important to note that our sample included a large number of African American Veterans, and that our data represented all Veterans seen in VA primary care. VA is a large, capitated, healthcare system, and further research should determine how applicable our findings are to other healthcare systems. Our data were drawn from administrative datasets, and may lack the granularity required for ascertaining disease severity and duration of mental illnesses.

The recent spotlight on the opioid epidemic is a reflection that SUDs are in part driving mortality among Americans. As shown in the Case and Deaton study, non-Hispanic White midlife mortality is being driven by increase in SUDs which may include Veterans. Yet, SUD is not considered a serviceconnected condition, and much of the attention on Veteran mental health has focused on post-traumatic stress disorder. Our study highlights that SUDs are deadly inside the VA just as they are deadly outside of the VA. Our study represents the largest study of life expectancy and common causes of death within the USA. Quantifying the life expectancy and causes of death among common mental illnesses is a necessary step in informing approaches for addressing the persistent and growing public health problem of mental illnesses. The study highlights the critical public health issue of mental illness and emphasizes that individuals with mental illnesses are at risk for premature mortality. Broadening the discussion of mental illness to include mortality risk can focus healthcare systems in better screening and treatment of mental illnesses especially in the presence of medical conditions. Our findings may spur public debate regarding the best policies that can improve the health outcomes and improve longevity among those with a mental illness.

Acknowledgments: Dr. Trivedi was supported by a VA HSR\&D CDA.

Corresponding Author: Ranak B. Trivedi, PhD; Center for Innovation to Implementation, VA Palo Alto Health Care System 795 Willow Rd 152-MPD, Menlo Park 94025, CA, USA (e-mail: ranak.trivedi@va. gov).

Funding Information: This work was financially supported by the VA National Primary Care Analytic and Evaluation Unit.

\section{Compliance with Ethical Standards:}

Disclaimer: The views expressed herein are those of the authors and do not reflect those of the Department of Veterans Affairs.

Conflict of Interest: The authors declare that they do not have a conflict of interest.

\section{REFERENCES}

1. Druss BG, Zhao L, Von Esenwein S, Morrato EH, Marcus SC. Understanding Excess Mortality in Persons with Mental Illness: 17-year Follow Up of a Nationally Representative US Survey. Med Care 2011;49(6):599-604.

2. Walker ER, McGee RE, Druss BG. Mortality in mental disorders and global disease burden implications: a systematic review and metaanalysis. JAMA Psychiat 2015;72(4):334-341. 
3. Newcomer JW, Hennekens CH. Severe Mental Illness and Risk of Cardiovascular Disease. JAMA. 2007;298(15):1794-1796.

4. Olfson M, Ascher-Svanum H, Faries DE, Marcus SC. Predicting Psychiatric Hospital Admission Among Adults with Schizophrenia. Psychiatr Serv 2011;62(10):1138-1145.

5. Druss BG, Walker ER. Mental Disorders and Medical Comorbidity. The Synthesis project Research Synthesis Report 2011;(21):1-26.

6. Pratt LA, Druss BG, Manderscheid RW, Walker ER. Excess mortality due to depression and anxiety in the United States: results from a nationally representative survey. Gen Hosp Psychiatry 2016;39:39-45.

7. Trivedi RB, Post EP, Sun H, et al. Prevalence, Comorbidity, and Prognosis of Mental Health Among US Veterans. Am $J$ Public Health 2015;105(12):2564-2569.

8. Case A, Deaton A. Rising morbidity and mortality in midlife among white non-Hispanic Americans in the 21st century. Proc Natl Acad Sci U S A 2015;112(49): 15078-15083.

9. Kilbourne AM, Morden NE, Austin K, et al. Excess heart-disease-related mortality in a national study of patients with mental disorders: identifying modifiable risk factors. Gen Hosp Psychiatry 2009;31(6):555-563.

10. Hoerster KD, Lehavot K, Simpson T, McFall M, Reiber G, Nelson KM. Health and Health Behavior Differences: U.S. Military, Veteran, and Civilian Men: Am J Prev Med 2012;43(5):483-489.

11. Zivin K, Yosef M, Miller EM, et al. Associations between depression and all-cause and cause-specific risk of death: a retrospective cohort study in the Veterans Health Administration. J Psychosom Res 2015;78(4):324331 .

12. Rosland AM, Nelson K, Sun H, et al. The patient-centered medical home in the Veterans Health Administration. Am $J$ Manag Care 2013; 19(7):e263-272.

13. CDW (n.d.) Corporate Data Warehouse. In: Department of Veterans Affairs.

14. Kim HM, Smith EG, Stano CM, et al. Validation of key behaviourally based mental health diagnoses in administrative data: suicide attempt, alcohol abuse, illicit drug abuse and tobacco use. BMC Health Serv Res 2012;12:18.

15. VIReC. Mortality Data. In: Department of Veterans Affairs. https://vaww. virec.research.va.gov/Index.htm. Accessed 11 Sept 2019.

16. Bossarte R. An overview of VA/DoD joint mortality data repository and data from studies conducted by the epidemiology program in VA's office of public health. In: VIReC Cyberseminar Archive. 2016.

17. Lee J, Kresina TF, Campopiano M, Lubran R, Clark HW. Use of Pharmacotherapies in the Treatment of Alcohol Use Disorders and Opioid Dependence in Primary Care. Biomed Res Int 2015;2015:137020.

18. Boggs JM, Simon GE, Ahmedani BK, Peterson E, Hubley S, Beck A. The Association of Firearm Suicide With Mental Illness, Substance Use Conditions, and Previous Suicide Attempts. Ann Intern Med 2017;167(4):287-288.

19. Olfson M, Gerhard T, Huang C, Crystal S, Stroup TS. Premature Mortality Among Adults with Schizophrenia in the United States. JAMA Psychiat 2015;72(12):1172-1181.

20. Gierisch JM, Nieuwsma JA, Bradford DW, et al. Pharmacologic and behavioral interventions to improve cardiovascular risk factors in adults with serious mental illness: a systematic review and meta-analysis. J Clin Psychiatry 2014;75(5):e424-440.

21. Zivin K, Pfeiffer PN, Szymanski BR, et al. Initiation of Primary CareMental Health Integration programs in the VA Health System: associations with psychiatric diagnoses in primary care. Med Care 2010;48(9):843-851.

Publisher's Note Springer Nature remains neutral with regard to jurisdictional claims in published maps and institutional affiliations. 